\title{
Run compressed rank/select for large alphabets
}

\section{Fuentes-Sepulveda, J.}

IEEE

2018

Fuentes-Sepulveda , J , Karkkainen , J , Kosolobov , D \& Puglisi , S 2018 , Run compressed rank/select for large alphabets . in A Bilgin , M W Marcellin , J Serra-Sagrista \& J A Storer pÿ(eds) , 2018 Data Compression Conference 2730 March 2018 Snowbird, Utah, USA . DCC , IEEE , pp. 315-324, Data Compression Conference, Snowbird, Utah, United States , 27/03/2018 . https://doi.org/10.1109/DCC.2018.00040

http://hdl.handle.net/10138/307236

https://doi.org/10.1109/DCC.2018.00040

other

acceptedVersion

Downloaded from Helda, University of Helsinki institutional repository.

This is an electronic reprint of the original article.

This reprint may differ from the original in pagination and typographic detail.

Please cite the original version. 


\title{
Run Compressed Rank/Select for Large Alphabets*
}

\author{
José Fuentes-Sepúlveda ${ }^{1}$, Juha Kärkkäinen ${ }^{2}$, Dmitry Kosolobov ${ }^{2}$, and Simon J. Puglisi² \\ ${ }^{1}$ Department of Computer Science, University of Chile, Santiago, Chile \\ ${ }^{2}$ Helsinki Institute for Information Technology, \\ Department of Computer Science, University of Helsinki, Helsinki, Finland
}

\begin{abstract}
Given a string of length $n$ that is composed of $r$ runs of letters from the alphabet $\{0,1, \ldots, \sigma-1\}$ such that $2 \leq \sigma \leq r$, we describe a data structure that, provided $r \leq n / \log ^{\omega(1)} n$, stores the string in $r \log \frac{n \sigma}{r}+o\left(r \log \frac{n \sigma}{r}\right)$ bits and supports select and access queries in $O\left(\log \frac{\log (n / r)}{\log \log n}\right)$ time and rank queries in $O\left(\log \frac{\log (n \sigma / r)}{\log \log n}\right)$ time. We show that $r \log \frac{n(\sigma-1)}{r}-O\left(\log \frac{n}{r}\right)$ bits are necessary for any such data structure and, thus, our solution is succinct. We also describe a data structure that uses $(1+\epsilon) r \log \frac{n \sigma}{r}+O(r)$ bits, where $\epsilon>0$ is an arbitrary constant, with the same query times but without the restriction $r \leq n / \log ^{\omega(1)} n$. By simple reductions to the colored predecessor problem, we show that the query times are optimal in the important case $r \geq 2^{\log ^{\delta} n}$, for an arbitrary constant $\delta>0$. We implement our solution and compare it with the state of the art, showing that the closest competitors consume $31-46 \%$ more space.
\end{abstract}

\section{Introduction}

Data structures supporting rank and select queries on sequences are fundamental to a wide variety of topics in the theoretical and practical computer science, especially as a component of more complex succinct and compressed data structures (we provide a formal definition of rank and select queries below). Rank and select structures for non-binary strings have been of interest since the advent of the FM-index [8] and the compressed suffix array [12], and subsequent works on other indexes based on the Burrows-Wheeler transform [5] (BWT) (e.g., see [15]).

The simple run-length encoding of the BWT of a string allows to achieve, on highly repetitive strings, compression ratios that are comparable to the compression ratios achieved by the best reference-based schemes such as LZ77 [19]. The crucial component required for the implementation of compressed indexes based on BWT is the support of the rank (and, sometimes, select) queries on the run-length encoded BWT. Many works have been published on this and related topics (e.g., see [2, 11, $15,16]$ and references therein), but none of them could achieve optimal (succinct) run-length compressed space and optimal time simultaneously (recall that a data

\footnotetext{
*This work is supported by the Academy of Finland via grant 1294143 and by the EU grant H2020-MSCA-RISE-2015 BIRDS690 No. 690941. The first author received funding from Fondecyt grant 3170534 and Basal Funds FB0001, Conicyt, Chile. Part of this work was developed during the Shonan Meeting 126 "Computation over Compressed Structured Data".
} 
structure is called succinct if it occupies $Z+o(Z)$ space, where $Z$ is the information theoretic lower bound for its size). This line of research became especially interesting after a recent paper by Gagie et al. [9], in which these authors proposed a method that allows to avoid the $O\left(\frac{n}{\operatorname{polylog}(n)}\right)$ bits of redundancy that were required in all previous BWT-based indexes supporting the full range of common search operations.

In this paper we describe a data structure that, given a string of length $n$ with $r$ runs of letters from the alphabet $\{0,1, \ldots, \sigma-1\}$ such that $2 \leq \sigma \leq r \leq n / \log ^{\omega(1)} n$, stores the string in $r \log \frac{n \sigma}{r}+o\left(r \log \frac{n \sigma}{r}\right)$ bits of space (for brevity, log denotes the logarithm with base 2), and supports select and access queries in $O\left(\log \frac{\log (n / r)}{\log \log n}\right)$ time

and rank queries in $O\left(\log \frac{\log (n \sigma / r)}{\log \log n}\right)$ time. Further, we prove that $r \log \frac{n(\sigma-1)}{r}-O\left(\log \frac{n}{r}\right)$ bits are necessary for any such encoding in the worst case and this implies that our data structure is succinct. We also describe a version of this data structure that uses $(1+\epsilon) r \log \frac{n \sigma}{r}+O(r)$ bits, for arbitrary constant $\epsilon>0$, with the same query times but without the restriction $r \leq n / \log ^{\omega(1)} n$. We then show, via reductions to the socalled colored predecessor problem, that provided $r \geq 2^{\log ^{\delta} n}$ for an arbitrary constant $\delta>0$, our rank, select, and access times are optimal, even if $\sigma=2$. We also describe a generic version of our solution with a parameter controlling time-space trade-offs.

We have implemented our data structure and experiments show that our closest competitors consume 31\%-46\% more space; this small space usage, however, comes at the price of a noticeable slowdown in query time on some inputs.

This paper is organized as follows. In the next section we first discuss some auxiliary tools and then describe the main data structure. The subsequent section is devoted to time and space optimality considerations. The last section presents a practical implementation of these ideas and experiments.

Preliminaries. Let $s$ be a string of length $n$. The letters of $s$ are denoted $s[0], s[1]$, $\ldots, s[n-1]$ and $s[i . . j]$ denotes $s[i] s[i+1] \cdots s[j]$. Our notation for arrays is similar: e.g., $a[0 . . n-1]$ is an array of length $n$. A run in $s$ is a substring $s[i . . j]$ in which all letters are equal. For any $i, j$, the set $\{k \in \mathbb{Z}: i \leq k \leq j\}$ is denoted $[i . . j]$.

For a string or an array $B$, the query $\operatorname{access}(i, B)$ merely returns $B[i]$, the query $\operatorname{rank}_{c}(i, B)$ returns the number of letters $c$ in $B[0 . . i]$, the query $\operatorname{select}_{c}(i, B)$ returns the position of the $i$ th letter $c$ in $B$ or returns -1 if either $i<1$ or $B$ contains less than $i$ letters $c$ (in particular, $\operatorname{select}_{c}(0, B)=-1$ ). We omit the second parameter $B$ and write simply $\operatorname{rank}_{c}(i), \operatorname{select}_{c}(i)$, access $(i)$ when $B$ is clear from the context.

\section{Data Structure}

We briefly describe the so-called Elias-Fano scheme [6, 7], which is of fundamental importance for succinct data structures and which we use in our construction below.

Elias-Fano scheme. Consider a bit array of length $n$ that contains exactly $k$ ones. In the Elias-Fano scheme we split the array into $k$ buckets of lengths $\left\lceil\frac{n}{k}\right\rceil$ (the last bucket can be smaller), concatenate the unary encodings of the numbers of ones in the buckets, thus obtaining a bit array $B$ of length $2 k$ (e.g., the bit array 001101 encodes three buckets containing, respectively, 2, 0, and 1 ones), and finally, store consecu- 
tively the relative positions of the ones inside the buckets in an array $A[0 . . k-1] . A$ occupies $k\left\lceil\log \frac{n}{k}\right\rceil$ bits, and the whole encoding takes $k \log \frac{n}{k}+O(k)$ bits in total. In addition, $B$ is equipped with the following data structure, adding $o(k)$ bits.

Lemma 1 (see [13]). Any bit array of length $n$ has an encoding that occupies $n+o(n)$ bits and supports the queries rank $_{0}$, rank $_{1}$, select , select $_{1}$ in $O(1)$ time.

Using the data structure of Lemma 1, one can compute the bucket containing the $i$ th one as $\operatorname{select}_{0}(i, B)-i+1$. The relative position of this one in the bucket is stored explicitly in $A[i]$. Therefore, any $\operatorname{select}_{1}(i)$ query on the bit array can be answered in $O(1)$ time. We further enhance this basic scheme as follows.

Lemma 2 (see [2, Th. 14]). Given a set $S \subset[0 . . u]$ of size $k$, there is a data structure that occupies $O\left(k \log \frac{u}{k}\right)$ bits and supports, for any given $x$, predecessor queries $\max \{y \in S: y<x\}$ in $O\left(\log \log _{w} \frac{u}{k}\right)$ time, where $w$ is the size of machine word.

Lemma 3. Let $\tau \geq 1$ be a "sampling" parameter. Any bit array of length $n$ containing exactly $k$ ones has an encoding that occupies $\left(1+\frac{1}{\tau}\right) k \log \frac{n}{k}+O(k)$ bits and supports select ${ }_{1}$ queries in $O(1)$ time and rank $_{0} /$ rank $_{1}$ queries in $O\left(\log \tau+\log \frac{\log (n / k)}{\log \log n}\right)$ time.

Proof. Since select ${ }_{1}$ was discussed above and $\operatorname{rank}_{0}(i)=i-\operatorname{rank}_{1}(i)+1$, it suffices to consider rank $_{1}$. Let $b=\lfloor i /\lceil n / k\rceil\rfloor+1$. Obviously, $i$ lies in the $b$ th bucket and, hence, $d=$ select $_{1}(b-1, B)-b+2$ ones from other buckets precede $i$. Thus, it remains to count the number of ones before position $i$ that also lie in the $b$ th bucket.

It is easy to see that there are $\ell=\operatorname{select}_{1}(b, B)-$ select $_{1}(b-1, B)-1$ ones in the $b$ th bucket and their relative positions are stored in the subarray $A[d . . d+\ell-1]$. Denote $\rho=c \tau$, where $c$ is a positive constant determined below. If $\ell \leq \rho$, we use binary search to count in $O(\log \rho)=O(\log \tau)$ time the number of subarray elements that precede the relative position of $i$ inside the $b$ th bucket, i.e., precede $i \bmod \lceil n / k\rceil$. For the case $\ell>\rho$, we sample every $\lceil\rho\rceil$ th element of the subarray and put them in the data structure from Lemma 2 occupying $O\left(\frac{k_{b}}{\rho}\left\lceil\log \frac{n}{k}\right\rceil\right)$ bits, where $k_{b}$ is the number of ones in the $b$ th bucket, which allows us to count the number of sampled predecessors of $i \bmod \lceil n / k\rceil$ in $O\left(\log \frac{\log (n / k)}{\log \log n}\right)$ time; the $\lceil\rho\rceil-1$ non-sampled elements following the found sampled predecessor are processed again by binary search. We store these data structures consecutively and locate the required one using an additional bit array of length $k$ that marks the sampled elements of $A$; the details are omitted as they are straightforward. The overall space can be estimated as $k \log \frac{n}{k}+O\left(k+\frac{k}{\rho} \log \frac{n}{k}\right)$, which is $\left(1+\frac{1}{\tau}\right) k \log \frac{n}{k}+O(k)$ for an appropriately chosen constant $c$ in $\rho=c \tau$.

For example, when $\tau=\log n$, Lemma 3 gives us a data structure that occupies $k \log \frac{n}{k}+O(k)$ bits and answers rank queries in $O(\log \log n)$ time.

The main data structure. Let us consider a string $s$ of length $n$ that can be represented as a concatenation of $r$ runs of letters from the alphabet $[0 . . \sigma-1]$ such that $2 \leq \sigma \leq r$. Denote by $n_{0}, n_{1}, \ldots, n_{\sigma-1}$ the number of runs of the letters $0,1, \ldots, \sigma-1$, respectively; note that $n_{0}+n_{1}+\cdots+n_{\sigma-1}=r$. For $c \in[0 . . \sigma-1]$ and $i \in\left[1 . . n_{c}\right]$, let $\ell_{c, i}$ denote the length of the $i$ th run of the letter $c$. We encode $s$ in the following components (see a clarifying example in Fig. 1): 
- a bit array $R[0 . . n-1]$ such that $R[i]=1$ iff $s[i] \neq s[i+1]$ or $i=n-1$;

- a string $H[0 . . r-1]$ such that $H[i]=s\left[\operatorname{select}_{1}(i+1, R)\right]$;

- a bit array $C[0 . . r+\sigma-1]$ that is the concatenation of the unary encodings for the number of runs of each letter;

- an integer array $S[0 . . r-1]$ that stores the following numbers (in this order): $\ell_{0,1}, \ell_{0,2}, \ldots, \ell_{0, n_{0}}, \ell_{1,1}, \ell_{1,2}, \ldots, \ell_{1, n_{1}}, \ldots, \ell_{\sigma-1,1}, \ell_{\sigma-1,2}, \ldots, \ell_{\sigma-1, n_{\sigma-1}}$.

Thus, $R$ marks the last letter of every run in $s, H$ stores these letters in the corresponding order, $C$ encodes the number of runs of each letter, and $S$ stores the run lengths grouped by letters.

Let us choose a positive "sampling" parameter $\rho \geq 1$ that will regulate time-space trade-offs for the data structure. Our goal is

$$
\begin{aligned}
s & =\text { aaaabbbadddddaaaaaddbaaaa, } \\
R & =0001001100001000010110001 \\
H & =\text { abadadba } \\
C & =000010011001 \\
S & =4,1,5,4,3,1,5,2 .
\end{aligned}
$$

Figure 1: Here $\sigma=4$ and, for the readability, $a, b, c, d$ denote the letters $0,1,2,3$. to support the queries select $c_{c}$ and access in $O\left(\rho+\log \frac{\log (n / r)}{\log \log n}\right)$ time and the query rank r $_{c}$ in $O\left(\rho+\log \frac{\log (n \sigma / r)}{\log \log n}\right)$ time (see optimality considerations below).

We encode $R$ in $\left(1+\frac{1}{2^{\rho}}\right) r \log \frac{n}{r}+O(r)$ bits as in Lemma 3 with $\tau=2^{\rho}$. The array $C$ is encoded in $O(r)$ bits as in Lemma 1. The string $H[0 . . r-1]$ is stored in the following data structure of Belazzougui and Navarro [2] (slightly reformulated).

Lemma 4 (see [2, Th. 6]). Let $\rho \geq 1$ be a "sampling" parameter. Any string of length $r$ over the alphabet $[0 . . \sigma-1]$ such that $2 \leq \sigma \leq r$ has an encoding that occupies $\left(1+\frac{1}{\rho}\right) r \log \sigma+o(r \log \sigma)+O(r)$ bits and supports access in $O(\rho)$ time, select $_{c}$ in $O(1)$ time, and rank $_{c}$ in $O\left(\log \frac{\log \sigma}{\log \log n}\right)$ time.

Proof. The result follows from the proof of [2, Theorem 6] if we put $f(n, \sigma)=\rho$.

The structures $R$ and $H$ are already sufficient to implement access queries in $O\left(\rho+\log \frac{\log (n / r)}{\log \log n}\right)$ time since $s[i]=H\left[\operatorname{rank}_{1}(i-1, R)\right]$. For rank $_{c}$ and select ${ }_{c}$, we need $C$ and $S$. It turns out that we do not have to store $S$ explicitly since, as it is shown below, $S[i]$ can be computed in $O(1)$ time, for any $i \in[0 . . r-1]$, using $R, H$, and $C$. However, besides access, our data structure requires to answer on $S$ the queries $\operatorname{pred}(x, S)$ that return the maximal $i \in[0 . . r-1]$ such that $S[0]+S[1]+\cdots+S[i]<x$.

In order to perform pred in small space and $O\left(\rho+\log \frac{\log (n / r)}{\log \log n}\right)$ time, we sample the numbers $S[0]+S[1]+\cdots+S[j\lceil\rho\rceil]$, for $j \in$ $\left[0 . . \frac{r-1}{\lceil\rho\rceil}\right]$, and store them in the data structure from Lemma 2, thus consuming $O\left(\frac{r}{\rho} \log \frac{n \rho}{r}\right)$ bits. To perform $\operatorname{pred}(x, S)$, we first find in $O\left(\log \frac{\log (n \rho / r)}{\log \log n}\right) \leq O\left(\rho+\log \frac{\log (n / r)}{\log \log n}\right)$ time the maximal $j$ such that $S[0]+S[1]+\cdots+$

Table 1: Component sizes.

\begin{tabular}{cc}
\hline & size in bits \\
\hline$R$ & $\left(1+\frac{1}{2^{\rho}}\right) r \log \frac{n}{r}+O(r)$ \\
$H$ & $\left(1+\frac{1}{\rho}\right) r \log \sigma+o(r \log \sigma)+O(r)$ \\
$C$ & $O(r)$ \\
$S$ & $O\left(\frac{r}{\rho} \log \frac{n \rho}{r}\right)$ \\
\hline
\end{tabular}


$S[j\lceil\rho\rceil]<x$ and, then, compute $S[j\lceil\rho\rceil+1], S[j\lceil\rho\rceil+2], \ldots, S[(j+1)\lceil\rho\rceil]$ in $O(\rho)$ time, hence finding the answer in an obvious way.

The sizes of the described data structures are summarized in Table 1. Before discussing the implementation of rank $_{c}$ and select $c_{c}$, let us explain how one can compute $S[i]$ in $O(1)$ time using $R, H$, and $C$.

It follows from the definition of $C$ that $S[i]$ stores the length of a run of the letter $c=\operatorname{rank}_{1}\left(\operatorname{select}_{0}(i+1, C), C\right)$. Further, it is straightforward that there are exactly $j=$ select $_{1}(c, C)-c+1$ runs of letters $0,1, \ldots, c-1$ and therefore, by definition, $S[j]$ stores the length of the leftmost run of $c$. Hence, $S[i]$ stores the length of the $k$ th run of $c$, where $k=i-j+1$; we compute $k$ in $O(1)$ time. Then, we find $k^{\prime}=\operatorname{select}_{c}(k, H)+1$ in $O(1)$ time (see Lemma 4). Clearly, the $k$ th run of $c$ is the $k^{\prime}$ th run (of all runs) and its length, which is equal to $S[i]$, can be calculated as $\operatorname{select}_{1}\left(k^{\prime}, R\right)-\operatorname{select}_{1}\left(k^{\prime}-1, R\right)$ in $O(1)$ time.

Consider the $\operatorname{select}_{c}(i, s)$ query. Put $j=\operatorname{select}_{1}(c, C)-c+1$. As above, $S[j]$ is the length of the leftmost run of the letter $c$ (if any). Let us find the maximal $k$ such that $S[j]+S[j+1]+\cdots+S[j+k-1]<i$; obviously, the $i$ th occurrence of $c$ (if any) must lie in the $(k+1)$ st run of $c$. As $k=\operatorname{pred}(S[0]+\cdots+S[j-1]+i, S)-j+1$, it suffices to show how to compute $t=S[0]+\cdots+S[j-1]$. We calculate $t$ by summing $S\left[j^{\prime}\lceil\rho\rceil+1\right]+S\left[j^{\prime}\lceil\rho\rceil+2\right]+\cdots+S[j-1]$, where $j^{\prime}=\lfloor(j-1) /\lceil\rho\rceil\rfloor$, with the $\left(j^{\prime}+1\right)$ st number sampled from $S$, which, by definition, is equal to $S[0]+S[1]+$ $\cdots+S\left[j^{\prime}\lceil\rho\rceil\right]$, all in $O\left(\rho+\log \frac{\log (n / r)}{\log \log n}\right)$ time. Further, the $(k+1)$ st run of $c$ exists iff $c=\operatorname{rank}_{1}\left(\operatorname{select}_{0}(j+k+1, C), C\right)$; we check this condition and return -1 if the run does not exist. Otherwise, we calculate the sum $t^{\prime}=S[0]+S[1]+\cdots+S[j+k-1]$ in $O\left(\rho+\log \frac{\log (n / r)}{\log \log n}\right)$ time in the same way as we computed $t$; then, the $i$ th occurrence of $c$ in the string $s$ must be the $p$ th letter, where $p=i-\left(t^{\prime}-t\right)$, of the $(k+1)$ st run of $c$; thus, we obtain $\operatorname{select}_{c}(i, s)=\operatorname{select}_{1}\left(\operatorname{select}_{c}(k+1, H), R\right)+p$.

Consider the $\operatorname{rank}_{c}(i, s)$ query. Put $j=\operatorname{select}_{1}(c, C)-c+1$. Again, $S[j]$ is the length of the leftmost run of the letter $c$ (if any). In $O\left(\rho+\log \frac{\log (n / r)}{\log \log n}\right)$ time we compute $m=\operatorname{rank}_{1}(i-1, R)$, which is the number of runs preceding the position $i$ (excluding the run containing $i$ ). Then, $k=\operatorname{rank}_{c}(m-1, H)$ of them are runs of $c ; k$ is computed in $O\left(\log \frac{\log \sigma}{\log \log n}\right)$ time by Lemma 4 . Thus, the total length of the runs of $c$ preceding the position $i$ can be calculated as $x=S[j]+S[j+1]+\cdots+S[j+k-1]$ in $O\left(\rho+\log \frac{\log (n / r)}{\log \log n}\right)$ time (as in select $c_{c}$ above). It remains to check whether the position $i$ itself is inside a run of $c$ : it is so iff $H[m]=c$. Accordingly, we return $x+\left(i-\operatorname{select}_{1}(m, R)\right)$ if $H[m]=c$ (here $i-\operatorname{select}_{1}(m, R)$ is the position of $i$ in the run), and $x$ otherwise.

Lemma 5. Let $\tau \geq 1$ be a "sampling" parameter. Any string of length $n$ with $r$ runs over the alphabet $[0 . . \sigma-1]$ such that $2 \leq \sigma \leq r$ has an encoding that occupies $\left(1+\frac{1}{\tau}\right) r \log \frac{n \sigma}{r}+o(r \log \sigma)+O(r)$ bits and supports the queries select $_{c}$ and access in $O\left(\tau+\log \frac{\log (n / r)}{\log \log n}\right)$ time and rank $_{c}$ in $O\left(\tau+\log \frac{\log (n \sigma / r)}{\log \log n}\right)$ time.

Proof. The space required for $S$ is $O\left(\frac{r}{\rho} \log \frac{n \rho}{r}\right)=O\left(\frac{r}{\rho} \log \frac{n}{r}+r \frac{\log \rho}{\rho}\right) \leq O\left(\frac{r}{\rho} \log \frac{n}{r}\right)+$ $O(r)$. Summing up the space bounds from Table 1, we obtain $\left(1+O\left(\frac{1}{\rho}\right)\right) r \log \frac{n \sigma}{r}+$ 
$o(r \log \sigma)+O(r)$ bits. Further, select $c_{c}$ and access run in $O\left(\rho+\log \frac{\log (n / r)}{\log \log n}\right)$ time; rank $_{c}$ takes $O\left(\rho+\log \frac{\log (n / r)}{\log \log n}+\log \frac{\log \sigma}{\log \log n}\right)$ time, which can be simplified as $O\left(\rho+\log \frac{\log (n \sigma / r)}{\log \log n}\right)$. Putting $\rho=c \tau$ for an appropriate constant $c$, we obtain the result.

Theorem 1. Any string of length $n$ with $r$ runs over the alphabet $[0 . . \sigma-1]$ such that $2 \leq \sigma \leq r \leq n / \log ^{\omega(1)} n$ has an encoding that occupies $r \log \frac{n \sigma}{r}+o\left(r \log \frac{n \sigma}{r}\right)$ bits and supports select ${ }_{c}$ and access in $O\left(\log \frac{\log (n / r)}{\log \log n}\right)$ time and rank $_{c}$ in $O\left(\log \frac{\log (n \sigma / r)}{\log \log n}\right)$ time.

Proof. The result follows from Lemma 5 with $\tau=\log \frac{\log (n / r)}{\log \log n} \operatorname{since}$, for $r \leq n / \log ^{\omega(1)} n$, we have $\tau=\omega(1)$ and $r=o\left(r \log \frac{n}{r}\right)$.

Theorem 2. Any string of length $n$ with $r$ runs over the alphabet $[0 . . \sigma-1]$ such that $2 \leq \sigma \leq r$ has an encoding that occupies $(1+\epsilon) r \log \frac{n \sigma}{r}+O(r)$ bits, where $\epsilon$ is an arbitrary positive constant, and supports select ${ }_{c}$ and access queries in $O\left(\log \frac{\log (n / r)}{\log \log n}\right)$ time and rank $_{c}$ queries in $O\left(\log \frac{\log (n \sigma / r)}{\log \log n}\right)$ time.

Proof. Since $o(r \log \sigma) \leq \frac{1}{2 \epsilon} r \log \frac{n \sigma}{r}$ for large enough $n$, the result follows from Lemma 5 with $\tau=\frac{1}{2 \epsilon}$; the big-O notation hides the additive constant $\frac{1}{\epsilon}$ in the time bounds.

Lemma 5 implies many other trade-offs that we do not discuss separately.

\section{Optimality}

Clearly there is a one-to-one correspondence between the set $T$ of all strings of length $n$ with $r$ runs over the alphabet $[0 . . \sigma-1]$ and the pairs $(R, H)$ such that $R[0 . . n-2]$ is a bit array with $r-1$ ones and $H[0 . . r-1]$ is a string such that $H[i] \neq H[i+1]$, for $i \in[0 . . r-2]$. Hence, the size of $T$ is $\left(\begin{array}{c}n-1 \\ r-1\end{array}\right) \sigma(\sigma-1)^{r-1}$. Since $\log \left(x-\frac{1}{r}\right) \geq \log x-\frac{2}{r}$ for any $x \geq 1$ and $r \geq 2$, we obtain $\log \left(\begin{array}{l}n-1 \\ r-1\end{array}\right) \geq(r-1) \log \frac{n-1}{r-1} \geq(r-1) \log \left(\frac{n}{r}-\frac{1}{r}\right) \geq(r-$ 1) $\left(\log \frac{n}{r}-\frac{2}{r}\right)=r \log \frac{n}{r}-O\left(\log \frac{n}{r}\right)$ and thus $\log |T| \geq r \log \frac{n}{r}-O\left(\log \frac{n}{r}\right)+\log \left((\sigma-1)^{r}\right)=$ $r \log \frac{n(\sigma-1)}{r}-O\left(\log \frac{n}{r}\right)$, which implies the following lower bound and that therefore the data structure of Theorem 1 is succinct.

Theorem 3. Any encoding of a string of length $n$ with $r$ runs over an alphabet of size $\sigma$ requires at least $r \log \frac{n(\sigma-1)}{r}-O\left(\log \frac{n}{r}\right)$ bits in the worst case.

Let us investigate the optimality of the query times provided in Theorems 1 and 2. For this, we use reductions to the well-known colored predecessor data structure, in which one is given a set of $r$ integers from the universe $[0 . . n]$ each of which is colored either in red or blue, and the query asks to find, for a given integer $x$, the color of the maximal $y$ from this set such that $y \leq x$. Our reductions resemble those from $[18$, section 7] but we, nevertheless, present them for completeness.

Lemma 6. Suppose that, for any binary string of length $n$ with $r$ runs, there is an encoding that occupies $O\left(r \log ^{O(1)} n\right)$ bits and supports rank $_{c}$, select ${ }_{c}$, and access queries in, respectively, $t_{r}, t_{s}$, and $t_{a}$ time. Then, there is a colored predecessor data structure that stores $r$ integers (colored in red or blue) from the universe $[0 . . n]$ in $O\left(r \log ^{O(1)} n\right)$ bits of space and supports the colored predecessor queries in $O\left(\min \left\{t_{r}, t_{s}, t_{a}\right\}\right)$ time. 
Proof. Let $x_{1}, x_{2}, \ldots, x_{r}$ be the integers stored in our colored predecessor data structure. Suppose $t_{a} \leq \min \left\{t_{r}, t_{s}\right\}$. We create a bit string $s[0 . . n]$ such that $s[i]=1$ iff the predecessor of $i$ is colored in red. Then, the colored predecessor queries can be answered in $O\left(t_{a}\right)$ time using our $O\left(r \log ^{O(1)} n\right)$-bit encoding and access queries.

Suppose $t_{r} \leq \min \left\{t_{s}, t_{a}\right\}$. Then, we store the colors of $x_{1}, x_{2}, \ldots, x_{r}$ in an array $c[0 . . r-1]$ and create a bit string $s[0 . . n]$ such that $s[i]=1$ iff $i=x_{j}$ for some $j \in[1 . . r]$. Thus, the colored predecessor query can be answered as $c\left[\operatorname{rank}_{1}(x, s)-1\right]$ in $O\left(t_{r}\right)$ time.

Finally, suppose $t_{s} \leq \min \left\{t_{r}, t_{a}\right\}$. We create again the array $c$ and create a bit string $s[0 . . n+r]$ such that $s[i]=1$ iff $i=x_{j}+j-1$ for some $j \in[1 . . r]$. Then, the colored predecessor query can be answered as $c\left[\operatorname{select}_{0}(x, s)-x\right]$ in $O\left(t_{s}\right)$ time.

Assuming $r \geq 2^{\log ^{\delta} n}$ for a constant $\delta>0$ and putting $n^{\prime}:=r, S:=r \log ^{O(1)} n$, $w:=\Theta(\log n), \ell:=\log n$ in the formula of Pătraşcu and Thorup [17] (we denote their $n$ by $n^{\prime}$ to distinguish it from our $\left.n\right)$, we obtain the lower bound $\Omega\left(\min \left\{\frac{\log r}{\log \log n}, \log \frac{\log (n / r)}{\log \log n}\right.\right.$, $\log \log n, \log \log n\})=\Omega\left(\log \frac{\log (n / r)}{\log \log n}\right)$, which holds, by Lemma 6 , for rank , select $_{c}$, and access in any data structure occupying $O\left(r \log ^{O(1)} n\right)$ bits. Combining this with the lower bound $\Omega\left(\log \frac{\log \sigma}{\log \log n}\right)$ from [2] for rank, we deduce the following theorem.

Theorem 4. Any data structure that stores a string of length $n$ with $r$ runs in $O\left(r \log { }^{O(1)} n\right)$ bits requires $\Omega\left(\log \frac{\log (n \sigma / r)}{\log \log n}\right)$ time for rank $_{c}$ and $\Omega\left(\log \frac{\log (n / r)}{\log \log n}\right)$ time for select $_{c}$ and access in the worst case, provided $r \geq 2^{\log ^{\delta} n}$ for a constant $\delta \in(0,1)$.

Theorem 4 implies that the data structures of Theorems 1 and 2 are time optimal whenever $r \geq 2^{\log ^{\delta} n}$, for an arbitrary positive constant $\delta \in(0,1)$.

\section{Experimental Evaluation}

We implemented our data structure and measured its practical performance relative to other rank and select data structures available in the Succinct Data Structures Library (SDSL) [10], including the data structures of: Golynski et al. (gmr) [11], Barbay et al. (ap) [1], and Mäkinen and Navarro (rlmn) [14]. The implementation of gmr uses $n \log \sigma+o(n \log \sigma)$ bits and supports access, rank, and select in, resp., $O(\log \log \sigma)$, $O(\log \log \sigma)$, and $O(1)$ time. The implementation of ap uses $n H_{0}+o(n)\left(H_{0}+1\right)$ bits and supports access, rank, and select in $O(\log \log \sigma)$ worst-case time or $O\left(\log H_{0}\right)$ average time. The implementation of $r \operatorname{lmn}$ uses $2 r(2+\log (n / r))+\sigma \log n+u$ bits, where $u$ is the space of an underlying rank/select structure over a sequence of length $r$, and supports access, rank, and select in, resp., $O\left(\log (n / r)+u_{a}\right), O\left(\log (n / r)+u_{a}+u_{r}\right)$, and $O\left(\log (n / r)+u_{s}\right)$ time, where $u_{a}, u_{r}$, and $u_{s}$ correspond to the access, rank, and select times of the underlying structure; we use ap as the underlying structure as it showed the best time-space trade-offs. Also, we tested the data structure of Belazzougui et al. ( $r l e)$ [4], which uses $(1+\gamma) r \log \frac{n \sigma}{r}+O(r)$ bits and supports access, rank, and select in $O\left(\frac{1}{\gamma} \log \frac{n}{r}\right)$ time, for any $\gamma \in(0,1)$; rle is similar to our solution $^{1}$ but it was implemented only for small alphabets (hence we could not apply

\footnotetext{
${ }^{1}$ We thank the reviewers for informing us about this data structure.
} 
it for all datasets). Additionally, we implement a simple construction of [3] (bcgpr): it uses $O(r \log n)$ bits and supports rank and select in $O(\log \log n)$ time (access was not considered originally). The construction contains a pair of predecessor data structures for each letter $c \in[0 . . \sigma-1]$ : the first predecessor structure stores the starting indexes of runs of $c$ and the second one stores the number of letters $c$ before the starting index of each run of $c$. Our implementation of bcgpr uses binary searches instead of the predecessor structures and additionally we store the bit array $R$ and the string $H$ of our solution, but without rank/select support for $H$, in order to support access; thus, access takes $O\left(\log \frac{\log (n / r)}{\log \log n}\right)$ time and rank/select queries take $O(\log r)$ time.

Implementation. Our solution implements $R$ using Elias-Fano sparse bit array from the SDSL; for $H$, we used the SDSL implementations of gmr and ap for large alphabets, and huff for small alphabets; $C$ was encoded as a plain bit array supporting rank and select in $O(1)$ time; finally, $S$ was stored as an integer array with samples to support pred queries via binary search over $S$. The queries access, rank, and select were implemented verbatim. In the experiments, we call our solution $f k_{k p}^{2}$.

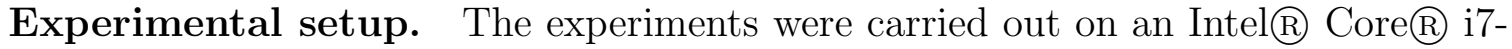
7700 machine with 4 physical cores, clocking at $3.6 \mathrm{GHz}$ each, with one $32 \mathrm{~KB} \mathrm{L1}$ instruction cache per core, one $32 \mathrm{~KB}$ L1 data cache per core, one $256 \mathrm{~KB}$ L2 cache per core, and one 8MB shared L3 cache. The code of all the structures was compiled with $\mathrm{g}^{++}$and optimization level -O3. The data structures were compared in terms of query times using the high-resolution $\mathrm{C}++$ function high_resolution_clock in the <chrono> library. The space consumption was measured by the serialization of data structures to their binary format. Experiments constructed each data structure on several datasets with varying $n$ and $\sigma$. We tested several power of two sampling steps for our structure and rle, but we report only 4, 16, and 32 since they exhibited the best time-space trade-offs. To differentiate each configuration, we use the name fkkp_x_y and rle_x to denote a sampling step of $\mathrm{x}$ and underlying structure $\mathrm{y}$.

The datasets are shown in Table 2. ${ }^{3}$ All Table 2: Datasets used in experiments. our datasets are the BWT of highly repetitive sequences. The datasets $w l_{-} 1 \mathrm{~B}$ and $\mathrm{w} \mathbf{l}_{-} 2 \mathrm{~B}$ were generated by taking the previous and the two previous symbols during the BWT computation of the sequence world-leaders from the PizzaECChili repetitive corpus ${ }^{4}$. Similarly, the datasets $\mathrm{kr} \_1 \mathrm{~B}$ and $\mathrm{kr} \_2 \mathrm{~B}$ were generated from

\begin{tabular}{lrrr}
\hline dataset & $n$ & $\sigma$ & runs \\
\hline wl_1B & $46,968,182$ & 90 & 573,487 \\
wl_2B & $46,968,182$ & 2,528 & 875,406 \\
wiki & $140,990,835$ & 174,796 & $2,586,752$ \\
kr_1B & $257,961,617$ & 161 & $2,791,368$ \\
kr_2B & $257,961,617$ & 7,124 & $4,194,799$ \\
\hline
\end{tabular}
the repetitive sequence kernel from Pizza\&Chili. The dataset wiki was generated from the edit history of some Wikipedia pages in which words were used as letters ${ }^{5}$.

\footnotetext{
${ }^{2}$ The implementation is available at https://github.com/jfuentess/sdsl-lite

${ }^{3}$ Available online at https://users.dcc.uchile.cl/ jfuentess/datasets/sequences.php

${ }^{4}$ http://pizzachili.dcc.uchile.cl/repcorpus. Last access: Nov. 2, 2017.

${ }^{5}$ https ://dumps . wikimedia.org/enwiki/20171001/enwiki-20171001-pages-meta-history10. xml-p3037476p3046511.7z. Last access: Nov. 2, 2017.
} 
Table 3: Space usage of the data structures in megabytes. Best results are underlined.

\begin{tabular}{|c|c|c|c|c|c|c|c|c|c|c|c|c|c|c|c|c|}
\hline & \multicolumn{3}{|c|}{ fkkp_gmr } & \multicolumn{3}{|c|}{ fkkp_ap } & \multicolumn{3}{|c|}{ fkkp_huff } & \multirow{2}{*}{ gmr } & \multirow{2}{*}{ ap } & \multirow{2}{*}{ rlmn } & \multirow{2}{*}{ bcgpr } & \multicolumn{3}{|c|}{ rle } \\
\hline & 4 & 16 & 32 & 4 & 16 & 32 & 4 & 16 & 32 & & & & & 4 & 16 & 32 \\
\hline wl_1B & 2.21 & 1.80 & 1.73 & 1.82 & 1.41 & 1.34 & 1.79 & 1.38 & 1.31 & 78.71 & 26.99 & 1.93 & 9.96 & 1.40 & 1.26 & $\underline{1.22}$ \\
\hline wl_2B & 4.15 & 3.53 & 3.42 & 3.24 & 2.61 & 2.51 & & & & 125.57 & 42.60 & 3.67 & 15.97 & & & \\
\hline wiki & 13.15 & 11.52 & 11.27 & 11.59 & 9.96 & 9.71 & & & & 402.42 & 274.61 & 12.75 & 53.56 & & & \\
\hline$k r_{-} 1 \mathrm{~B}$ & 10.55 & 8.55 & 8.22 & 8.47 & 6.47 & $\underline{6.14}$ & 8.53 & 6.54 & 6.20 & 440.30 & 230.83 & 8.84 & 48.29 & 7.18 & 6.53 & 6.40 \\
\hline kr_2B & 19.79 & 16.79 & 16.29 & 15.43 & 12.44 & 11.94 & & & & 698.00 & 410.50 & 16.21 & 76.43 & & & \\
\hline
\end{tabular}

Table 4: Running times for the access, rank, and select queries in $\mu$ s. Best times are underlined.

\begin{tabular}{|c|c|c|c|c|c|c|c|c|c|c|c|c|c|c|c|}
\hline & \multicolumn{5}{|c|}{ access } & \multicolumn{5}{|c|}{ rank } & \multicolumn{5}{|c|}{ select } \\
\hline & wl_1B & wl_2B & wiki & $k r_{-} 1 \mathrm{~B}$ & $\mathrm{kr} \_2 \mathrm{~B}$ & wl_1B & wl_2B & wiki & $\mathrm{kr}_{-} 1 \mathrm{~B}$ & $\mathrm{kr} \_2 \mathrm{~B}$ & |wl_1B & wl_2B & wiki & $\mathrm{kr} \_1 \mathrm{~B}$ & $\mathrm{kr}_{-} 2 \mathrm{~B}$ \\
\hline fkkp_gmr_4 & .18 & .49 & .66 & .26 & 1.03 & .86 & 2.04 & 2.02 & .99 & 2.64 & .92 & 1.32 & 1.30 & .99 & 1.45 \\
\hline fkkp_gmr_16 & .18 & .49 & .65 & .26 & 1.03 & 2.16 & 4.62 & 3.71 & 2.12 & 4.85 & 2.18 & 3.57 & 3.02 & 2.08 & 3.48 \\
\hline fkkp_gmr_32 & .18 & .49 & .64 & .26 & 1.03 & 3.42 & 6.61 & 5.81 & 3.50 & 7.58 & 3.89 & 6.49 & 5.20 & 3.52 & 6.16 \\
\hline fkkp_ap_4 & .26 & .42 & .64 & .32 & .62 & 1.15 & 1.54 & 2.82 & 1.39 & 2.73 & 1.23 & 1.66 & 3.03 & 1.57 & 2.78 \\
\hline fkkp_ap_16 & .26 & .42 & .64 & .32 & .62 & 3.62 & 3.93 & 6.88 & 3.82 & 6.69 & 3.06 & 4.22 & 6.84 & 3.76 & 6.31 \\
\hline fkkp_ap_32 & .26 & .42 & .63 & .32 & .62 & 5.64 & 6.61 & 11.49 & 6.79 & 11.38 & 5.51 & 7.50 & 11.45 & 6.63 & 10.98 \\
\hline fkkp_huff_4 & .11 & & & .17 & & .68 & & & .84 & & .88 & & & 1.11 & \\
\hline fkkp_huff_16 & .11 & & & .17 & & 2.14 & & & 2.42 & & 2.17 & & & 2.54 & \\
\hline fkkp_huff_32 & .11 & & & .17 & & 3.48 & & & 4.37 & & 3.93 & & & 4.47 & \\
\hline $\mathrm{gmr}$ & .16 & 1.81 & 2.52 & .29 & 2.45 & .27 & .74 & .60 & .36 & .45 & .30 & .48 & .66 & .91 & .50 \\
\hline ap & .34 & .62 & 1.22 & .57 & 1.07 & .16 & .23 & .48 & .31 & .39 & .68 & 1.23 & 1.50 & 2.75 & 3.38 \\
\hline rlmn & .26 & .42 & .63 & .32 & .62 & .49 & .73 & 1.17 & .59 & 1.10 & .54 & .79 & .75 & 1.52 & 1.58 \\
\hline bcgpr & .02 & .03 &.$\underline{03}$ & .03 & .03 & .19 & .17 & .31 & .32 & .30 & .12 & .11 & .19 & .19 & .16 \\
\hline $\mathrm{rle}_{4} 4$ & .41 & & & .56 & & .46 & & & .62 & & .52 & & & .69 & \\
\hline rle_16 & .93 & & & 1.23 & & .97 & & & 1.29 & & 1.08 & & & 1.39 & \\
\hline rle_32 & 1.58 & & & 2.07 & & 1.61 & & & 2.10 & & 1.74 & & & 2.28 & \\
\hline
\end{tabular}

Results. Table 3 shows the size of each data structure for all the datasets. In our experiments, the structure $\mathbf{f k p}$ _ap_32 provides the best space consumption, except for the dataset wl_1B, where rle_32 has the best consumption. For the underlying structures gmr, ap, and huff, our structure reduces its size by increasing the size of the sampling step. For small alphabets, we are comparable with rle. For large alphabets, the closest competitor, rlmn, uses from $31 \%$ to $46 \%$ more space than fkkp_ap_32.

Table 4 shows the query time of individual access, rank, and select queries. For each type of query, we executed 1,000,000 random queries, reporting the median time of an individual query achieved over ten non-consecutive executions. For access query, the best times were reached by bcgpr, being 10 times faster. The explanation is that while bcgpr performs an access to a plain sequence during the access query, the other structures need to perform an access over a succinct representation of a sequence. Notice that our structure has similar query time than the other structures. For rank query, the best times were reached by bcgpr for large alphabets and by ap for small alphabets. For small alphabets our structure is at most 5 times slower than the best competitor, using samplings steps of 4 . For large alphabets, the difference increases to at most 9 times. For the case of select queries, the best times were reached by bcgpr. In the worst result, dataset w1_2B, our structure is 12 times slower. In the best result, dataset wiki, our structure is 3.4 times slower. In general, for larger sampling steps, the query time of our structure increases. However, for larger sampling steps the size of our structure decreases. Thus, for large enough sequences with runs, our structure 
could maintain the sequences in main memory, meanwhile the other structure should access the disk, increasing their query time.

According to our experimental study, the best trade-offs of our structure are reached with the underlying structure ap and samplings steps of 16 or 32, if our focus is space, or with gmr and samplings of 4 or 16 , if our focus is query time.

\section{References}

[1] J. Barbay, F. Claude, T. Gagie, G. Navarro, and Y. Nekrich. Efficient fully-compressed sequence representations. Algorithmica, 69(1):232-268, 2014.

[2] D. Belazzougui and G. Navarro. Optimal lower and upper bounds for representing sequences. ACM Transactions on Algorithms, 11(4):1-21, 2015.

[3] D. Belazzougui, F. Cunial, T. Gagie, N. Prezza, and M. Raffinot. Composite repetitionaware data structures. In Proc. CPM, pages 26-39. Springer, 2015.

[4] D. Belazzougui, F. Cunial, T. Gagie, N. Prezza, and M. Raffinot. Flexible indexing of repetitive collections. In Proc. CiE, pages 162-174. Springer, 2017.

[5] M. Burrows and D. J. Wheeler. A block-sorting lossless data compression algorithm. Technical Report 124, Digital Equipment Corporation, Palo Alto, California, 1994.

[6] P. Elias. Efficient storage and retrieval by content and address of static files. Journal of the ACM, 21(2):246-260, 1974.

[7] R. M. Fano. On the number of bits required to implement an associative memory. Technical Report 61, Computer Structures Group, MIT, Cambridge, MA, 1971.

[8] P. Ferragina and G. Manzini. Opportunistic data structures with applications. In Proc. FOCS, pages 390-398. IEEE, 2000.

[9] T. Gagie, G. Navarro, and N. Prezza. Optimal-time text indexing in BWT-runs bounded space. In Proc. SODA, pages 1459-1477. SIAM, 2018.

[10] S. Gog, T. Beller, A. Moffat, and M. Petri. From theory to practice: Plug and play with succinct data structures. In Proc. SEA, pages 326-337. Springer, 2014.

[11] A. Golynski, J. I. Munro, and S. S. Rao. Rank/select operations on large alphabets: a tool for text indexing. In Proc. SODA, pages 368-373. SIAM, 2006.

[12] R. Grossi, A. Gupta, and J. S. Vitter. High-order entropy-compressed text indexes. In Proc. SODA, pages 841-850. ACM/SIAM, 2003.

[13] G. Jacobson. Space-efficient static trees and graphs. In Proc. FOCS, pages 549-554. IEEE, 1989.

[14] V. Mäkinen and G. Navarro. Succinct suffix arrays based on run-length encoding. Nordic Journal of Computing, 12(1):40-66, 2005.

[15] V. Mäkinen and G. Navarro. Compressed full-text indexes. ACM Computing Surveys, 39(1):2-62, 2007.

[16] V. Mäkinen, G. Navarro, J. Sirén, and N. Välimäki. Storage and retrieval of highly repetitive sequence collections. Journal of Computational Biology, 17(3):281-308, 2010.

[17] M. Pătraşcu and M. Thorup. Time-space trade-offs for predecessor search. In Proc. STOC, pages 232-240. ACM, 2006.

[18] R. Raman, V. Raman, and S. S. Rao. Succinct indexable dictionaries with applications to encoding k-ary trees, prefix sums and multisets. ACM Transactions on Algorithms, $3(4): 43: 1-43: 25,2007$.

[19] J. Ziv and A. Lempel. A universal algorithm for sequential data compression. IEEE Transactions on Information Theory, 23(3):337-343, 1977. 